\title{
Helmet to head coupling by multi-body system
}

\author{
L. Hynčík ${ }^{a, *}$, T. Bońkowski ${ }^{a}$ \\ ${ }^{a}$ NTIS - New Technologies for the Information Society, Faculty of Applied Sciences, University of West Bohemia, Technická 8, Pilsen, \\ Czech Republic
}

Received 7 February 2018; accepted 3 June 2019

\begin{abstract}
The number of fatalities proves that traffic accidents and their consequences are still a serious problem to be solved. Almost half of the people dying on the world's roads are pedestrians, cyclists, and two-wheeler riders, which are together called vulnerable road users. Future challenges such as multi-modal transport including autonomous cars and other modes of transport bring additional safety issues to be investigated. New impact scenarios, covering multi-directional impacts, can be hardly assessed using hardware dummies. Hence, the numerical simulation using human body models is the technology to be addressed for future safety assessment. Especially for vulnerable road users, the complex dynamical loading must be taken into account. Long impact scenarios with complex loading usually cost a lot of calculation time. The presented work contributes to the numerical assessment of two-wheeler riders' safety with a further view of the optimization of personal protective equipment. For running plenty of impact scenarios for optimization of the personal protective equipment, the short calculation time is necessary. The paper presents a multibody model of a helmeted two-wheeler rider, which results in a short calculation time for assessing complex impact scenarios. The previously developed and validated human body model is addressed and it is coupled to a helmet model using the multibody system approach. The helmeted human body model is validated in oblique impact scenarios by comparing the results to an existing finite element human body model.
\end{abstract}

(c) 2019 University of West Bohemia. All rights reserved.

Keywords: motorcycle, rider, helmet, coupling, multi-body system

\section{Introduction}

World Health Organization (WHO) monitors that every year the lives of approximately 1.25 million people are cut short as a result of a road traffic crash [14]. Moreover, between 20 and 50 million people suffer non-fatal injuries resulting in various types of disabilities. Nearly one half of those dying on the world's roads are so-called vulnerable road users (VRU): pedestrians, cyclists, and motorcyclists including two-wheeler (TW) riders [4].

Biomechanical human body models (HBM) play an important role in assessing the injuries caused by traffic accidents. The models complement hardware testing, especially for optimization procedures, where a lot of scenarios must be considered, or for scenarios, which are hard to be tested by an experimental approach. HBM could also augment the testing procedure for scenarios with non-standardized occupant anthropometry $[1,10]$.

The models can serve either for assessing the injury criteria due to the impact or for optimizing active and passive protective equipment (PPE) or devices. For assessing the injury criteria due to the traffic accidents of a passengers' car by the numerical approach, standard virtual human body models can be used. However, for the traffic modes, where a road user is comparable (by his/her size or by his/her mass) to the other vehicle, a complex loading scenario has to

*Corresponding author. Tel.: +420 377634 709, e-mail: hyncik@ntc.zcu.cz. https://doi.org/10.24132/acm.2019.432 
be investigated. The scenarios might also address coupling of HBM to PPE. For TW safety, a helmet is a typical PPE [6]. For such scenarios, a multibody system (MBS) approach for modeling the long scenario is the most suitable tool for the reduction of computational time. Since the optimization process concerns running plenty of simulations, the MBS approach for human body modeling is convenient to save the simulation time, as well.

The PPE is usually not compulsory to be wear by the TW riders. The only compulsory protective part is a helmet for the TW riders, but only in some countries [12]. Hence, for the investigation of various TW impact scenarios and for the PPE optimization, a helmet shall be an integral part of the TW rider model. The PPE is usually based on the energy absorption approach to mitigate the injury risk. So the numerical models of the PPE are usually based on the finite element (FE) method in order to ensure its deformability to absorb the energy.

The helmet is one of the most important protective equipment and it usually accommodates complex materials to ensure impact energy absorption and to influence the complex head/neck kinematics. In situations, where the helmet is not under optimization [8] and serves only for completing the model, it might be simplified to save the calculation time. However, the proper performance of the entire head/helmet system must be guaranteed.

\section{Method}

The current work couples the existing scalable hybrid human body Virthuman [7,13] to a simple helmet model by extending the MBS by adding the helmet as an additional rigid body. The simplification is schematically displayed in Fig. 1.
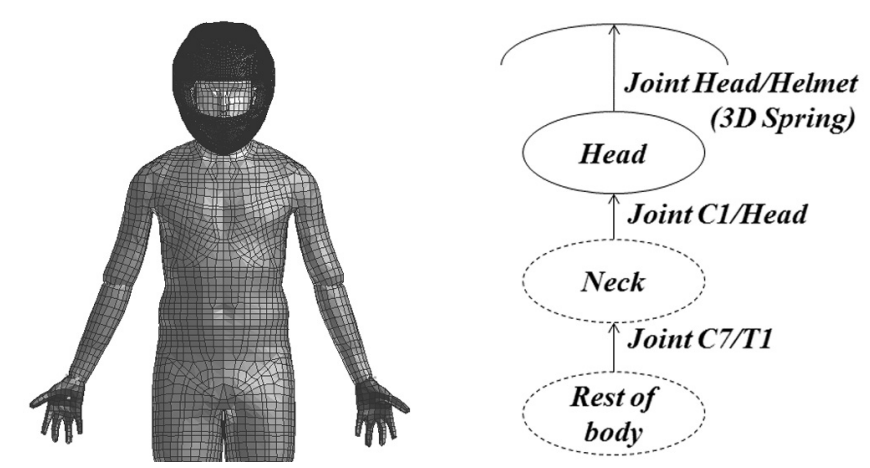

Fig. 1. Graphical visualization of the helmeted Virthuman model (left) and the scheme of the extended multibody system (right)

The head is linked to the helmet by a 3D nonlinear spring joint defined by stiffnesses in three independent orthogonal directions. The properties of this joint are developed based on the impacts using the full FE helmet model coupled to the Virthuman head in 4 major impact directions using several velocity levels. At the current stage, the rotational motion is avoided by high rotational stiffness and only the translational behavior is assumed. This assumption is supported by the argument that a considerable rotation between the head and the helmet is not expected in the reality. Since there is only the translational relative motion between the head and the helmet, the location of the 3D nonlinear spring joint does not affect the response and it is located in the head centre of gravity. The whole helmet/human MBS model is validated in oblique impact scenarios by means of the head acceleration and head helmet to ground contact force responses. 


\subsection{Impact scenarios}

The standards currently adopted around the world (ECE R22.05, SNELL 2010) [3] assess the helmet protective performance by a series of impacts. There is a metal headform in the helmet and every motorcycle helmet is subjected to four impacts: frontal, top, back and side. In all 4 cases, the peak of linear acceleration at the head centre of gravity has to be below a given limit of $275 \mathrm{~g}$ resulting in a $20 \%$ probability of skull fracture [11]. At the same time, the impact duration is also measured, so that high acceleration values can affect the headform only for limited durations. These measurements (the acceleration and the time) also define the head injury criterion (HIC), which passes or fails the threshold that is set to 2400 [3].

All 4 scenarios mentioned above are reconstructed firstly using an existing medium size FE helmet model accommodating a composite shell, an expanded polystyrene liner and a chin strap [5,6]. Fig. 2 shows the impact directions.

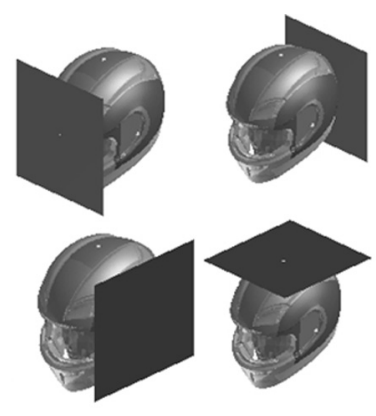

Fig. 2. Helmet impact directions

The helmet was positioned to cover the head, see Fig. 1 (left). For running the impacts, the head was extracted from the whole Virthuman model. For the interaction between the head and the helmet, there was a sliding contact with the contact friction equal to 0.5 as defined in the literature [9]. To fix the helmet from below, the chin strap was tightened under the chin [6].

Each impact scenario is reconstructed using 4 energy levels defined by impact velocities equal to $2.5 \mathrm{~m} / \mathrm{s}, 5 \mathrm{~m} / \mathrm{s}, 7.5 \mathrm{~m} / \mathrm{s}$ and $10 \mathrm{~m} / \mathrm{s}$, which provides the validity of the simplification for various impact directions. The setup configurations are in line with the experimental tests [6], which are used for the comparison between the simplified and the full FE helmet models.

\subsection{Helmet simplification}

For each impact direction and each impact velocity, the contact force depending on the helmet deformation is monitored. Putting all 4 impact responses for a particular impact direction together, the overall force/deformation curve is developed.

The simplified helmet model is developed using only the composite shell, which is converted to a rigid body. It serves as a contact surface. This rigid body is linked to the Virthuman MBS structure as an additional rigid body extending the head, see Fig. 1 (right). The link is modeled by a 3D nonlinear spring joint having the force/deformation characteristics adopted from the full FE helmet impacts. The spring did not limit the movement of the helmet shell to only translational displacement, the rotational displacement between the head and the helmet shell was fixed at this stage, where this assumption is approved by validation.

The main energy absorption part in the helmet is the liner. It contains a nonlinear foam material [6]. Such behavior is also reconstructed using the full FE helmet model with the Virthuman head loaded in 4 impact directions as defined in Fig. 2. The results for the frontal/back and side/top impacts are shown in Fig. 3 and Fig. 4, respectively. Each impact direction response is analyzed for 4 impact velocities equal to $2.5 \mathrm{~m} / \mathrm{s}, 5 \mathrm{~m} / \mathrm{s}, 7.5 \mathrm{~m} / \mathrm{s}$ and $10 \mathrm{~m} / \mathrm{s}$. 

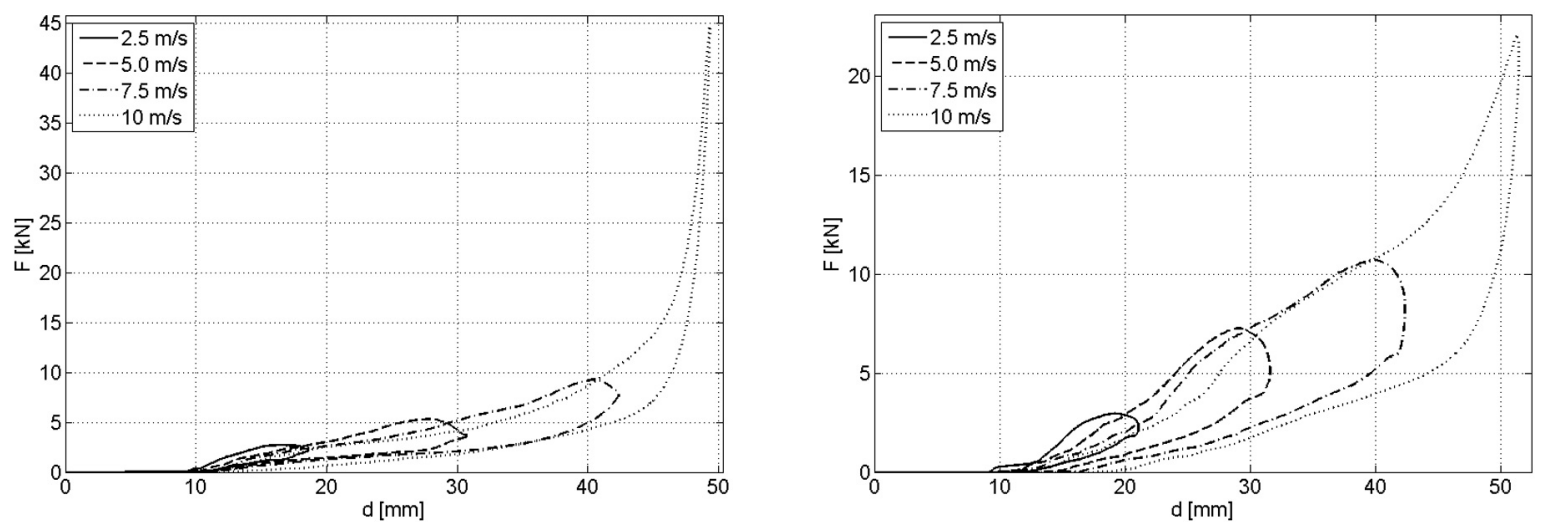

Fig. 3. Impact velocity dependent compression in the frontal (left) and back (right) directions
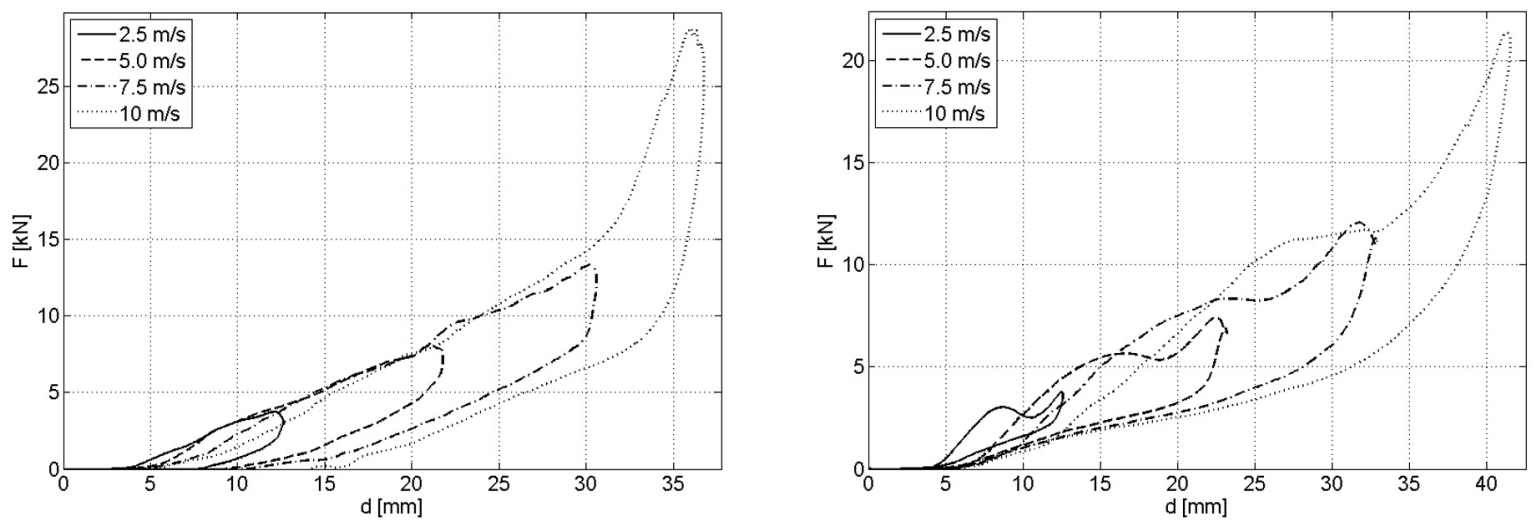

Fig. 4. Impact velocity dependent compression in the side (left) and top (right) directions

Figs. 3 and 4 summarize the peak forces for each impact direction and for every impact velocity. The pictures also show the nonlinear behavior with the increasing velocity. This situation allows to implement the helmet compression behavior into a single 3D nonlinear spring joint with 3 translational degrees of freedom. The frontal and back impacts correspond to the nonlinear response in the $x$-axis direction, the side impact corresponds to the nonlinear response in the $y$-axis and the top impact corresponds to the $z$-axis response. The nonlinear 3D spring joint stiffness for each degree of freedom is developed by connecting the peak values in each of 4 directions by the nonlinear response curve $F_{i}=F_{i}(i)$, where $i \in\{x, y, z\}$, see Fig. 5. Each translational degree of freedom absorbs the energy during the uploading phase by an exponential path. As discussed above, the three rotational degrees of freedom are fixed.

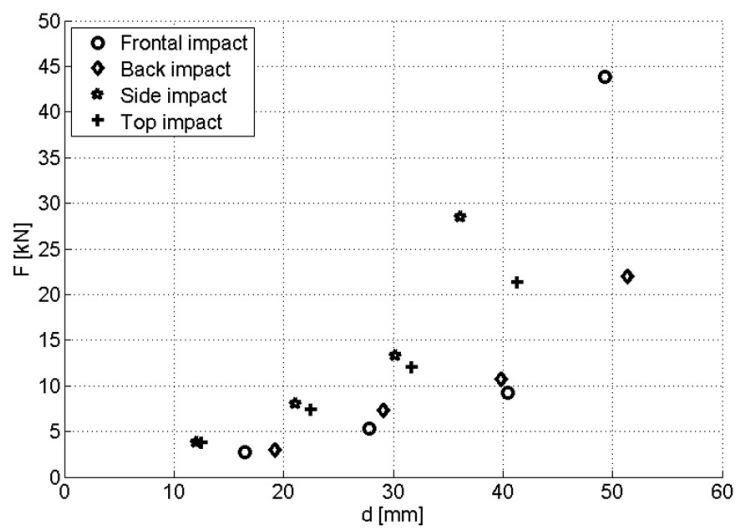

Fig. 5. Peak compression forces for 4 impact directions and for 4 impact velocities 


\subsection{Full model validation}

In most motorcycle accidents, the body axis angle towards the ground is usually $30^{\circ}$ or less [2]. Therefore, the simplified solution is tested in the oblique impacts according to the literature [6], where the comparison to the full validated FE model THUMS [12] is provided for comparison to the Virthuman model. The THUMS model is used because it has already been validated for the oblique impact scenarios including the helmet [6]. The model is loaded by the gravity considering different normal impact velocity $v_{n}$ and tangential (side) impact velocity $v_{t}$ (see Fig. 6).

In order to obtain comparable results, the Virthuman model is scaled [7] to the height of $175 \mathrm{~cm}$ and to the mass of $77 \mathrm{~kg}$ in order to correspond to the anthropometry of the THUMS model.

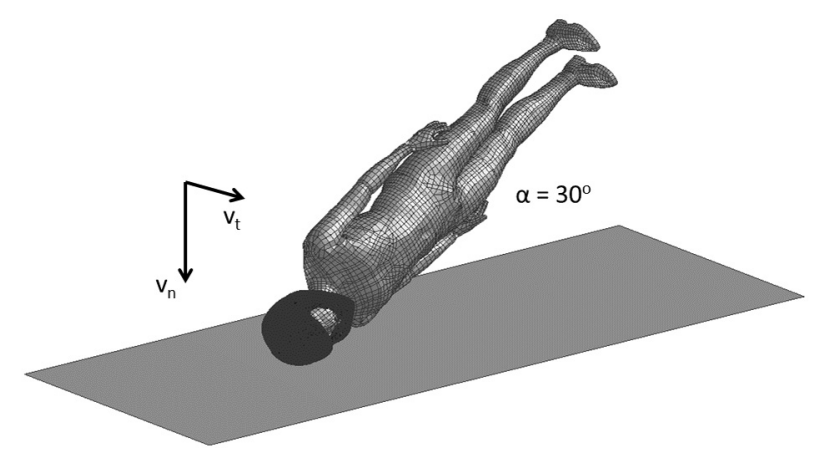

Fig. 6. Side impact scenario - full model validation

\section{Results}

The simplified helmet model performance is validated based on the oblique ground impacts combining the normal and the tangential velocities [6]. The combination of the normal and the tangential velocities brings complex kinematics of the head and the neck after the ground impact. The performance of the helmeted (both full FE and simplified helmet models) scaled virtual human body model Virthuman in the oblique impacts is compared also to the THUMS model by means of the relation of the maximum tangential and normal contact forces $\left(F_{t, \max } / F_{n, \max }\right)$ and the maximum head acceleration $a_{\max }$ [6]. The comparison between the performance of the full FE helmet model and the simplified one is shown in Figs. 7-9 for the particular combinations of the normal and the tangential velocities.
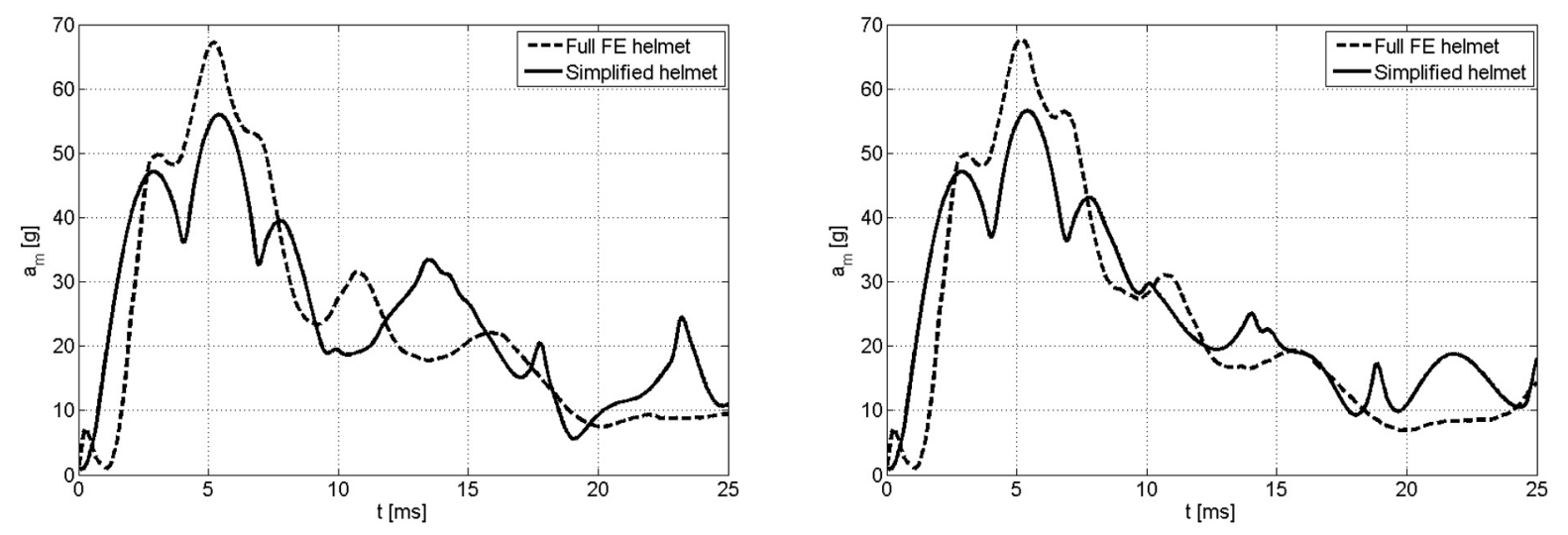

Fig. 7. Head acceleration resultant for $v_{n}=2.5 \mathrm{~m} / \mathrm{s}$ with $v_{t}=5 \mathrm{~m} / \mathrm{s}$ (left) and $v_{t}=10 \mathrm{~m} / \mathrm{s}$ (right) 

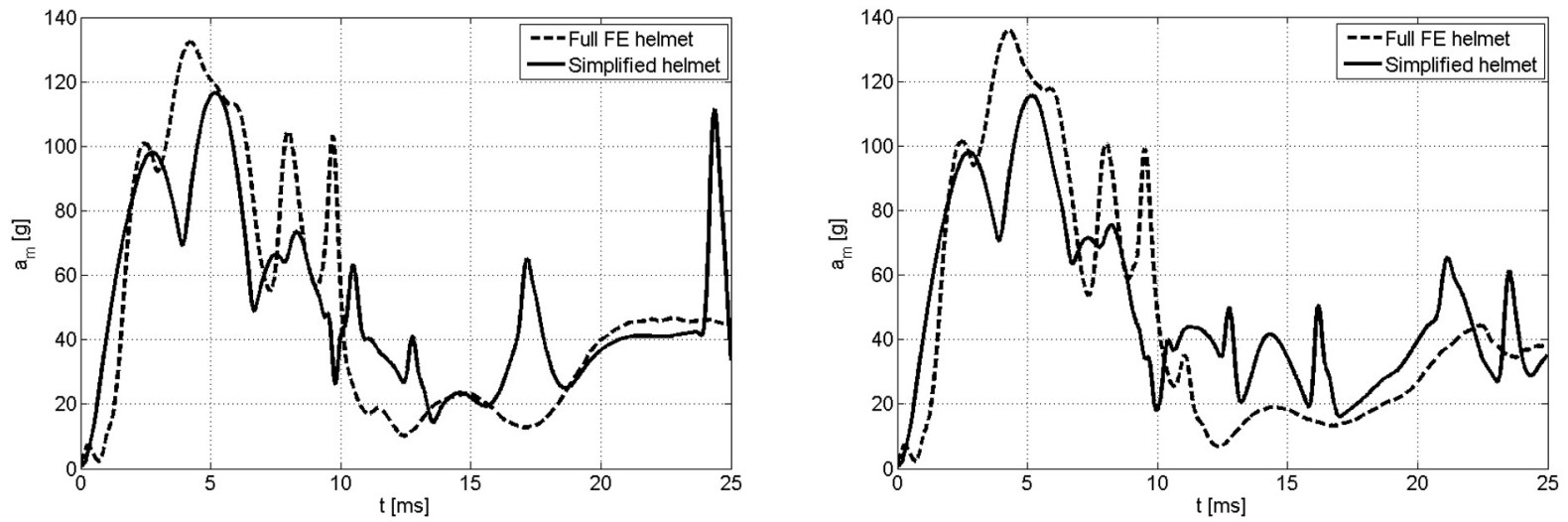

Fig. 8. Head acceleration resultant for $v_{n}=5 \mathrm{~m} / \mathrm{s}$ with $v_{t}=5 \mathrm{~m} / \mathrm{s}$ (left) and $v_{t}=10 \mathrm{~m} / \mathrm{s}$ (right)
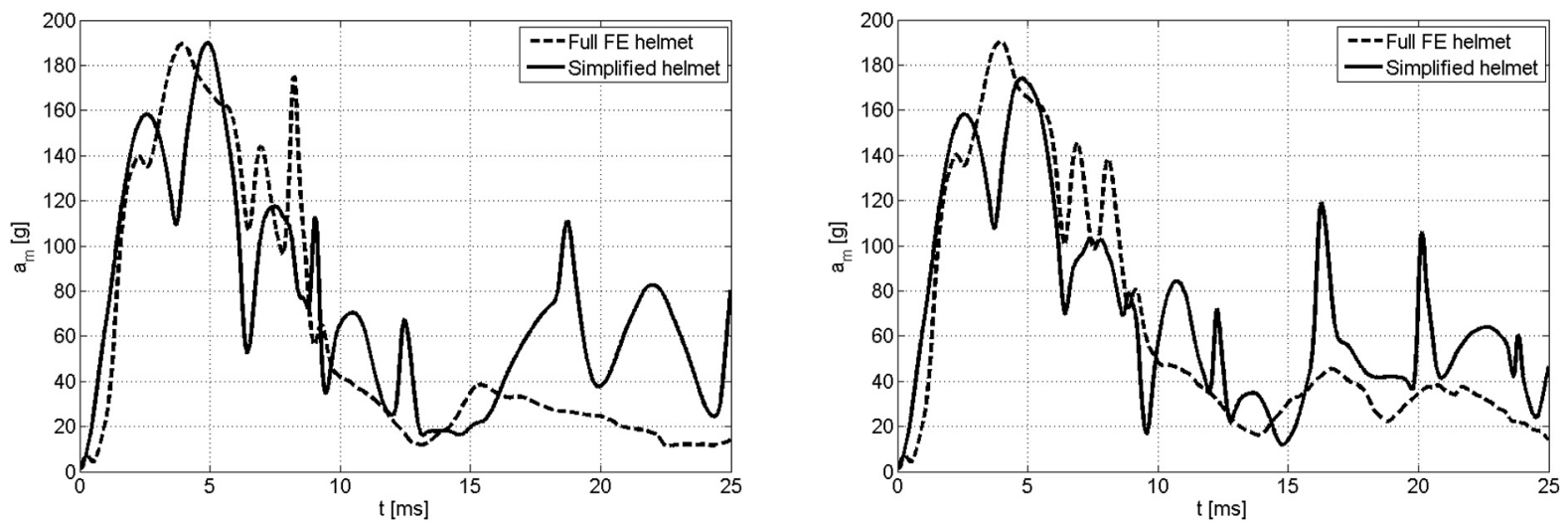

Fig. 9. Head acceleration resultant for $v_{n}=7.5 \mathrm{~m} / \mathrm{s}$ with $v_{t}=5 \mathrm{~m} / \mathrm{s}$ (left) and $v_{t}=10 \mathrm{~m} / \mathrm{s}$ (right)

Table 1 compares the three models by means of the ratio between the peak tangential and normal contact forces and by means of the peak head accelerations, namely the full FE helmeted Virthuman model, the Virthuman model with the simplified helmet and the full FE helmeted THUMS model. The Virthuman model shows a good correlation to the THUMS model for the normal impact velocity $v_{n}=2.5 \mathrm{~m} / \mathrm{s}$ and $5 \mathrm{~m} / \mathrm{s}$. For the normal impact velocity $v_{n}=7.5 \mathrm{~m} / \mathrm{s}$, the difference in the contact forces is higher than $30 \%$, which is probably caused by the simplified helmet model. This might be a limitation for exploiting the simplified approach in the case

Table 1. Comparison of the ratio between the peak tangential and normal contact forces as calculated by using the simplified helmet model (MB), the full FE helmet model and the helmeted THUMS (TH)

\begin{tabular}{|c|c|c|c|c|c|c|c|c|c|c|c|}
\hline \multicolumn{2}{|c|}{$\begin{array}{l}\text { Impact } \\
\text { velocity }\end{array}$} & \multicolumn{5}{|c|}{$\begin{array}{c}\text { Contact force } \\
F_{t, \text { max }} / F_{n, \text { max }}[-]\end{array}$} & \multicolumn{5}{|c|}{$\begin{array}{c}\text { Head acceleration } \\
a_{\max }[\mathrm{g}]\end{array}$} \\
\hline $\begin{array}{c}v_{n} \\
{[\mathrm{~m} / \mathrm{s}]}\end{array}$ & $\begin{array}{c}v_{t} \\
{[\mathrm{~m} / \mathrm{s}]}\end{array}$ & $\mathrm{MB}$ & $\mathrm{FE}$ & $\mathrm{TH}$ & $\begin{array}{c}\mathrm{MB} / \mathrm{FE} \\
{[\%]}\end{array}$ & $\begin{array}{c}\mathrm{MB} / \mathrm{TH} \\
{[\%]}\end{array}$ & $\mathrm{MB}$ & FE & $\mathrm{TH}$ & $\begin{array}{c}\mathrm{MB} / \mathrm{FE} \\
{[\%]}\end{array}$ & $\begin{array}{c}\mathrm{MB} / \mathrm{TH} \\
{[\%]}\end{array}$ \\
\hline \multirow{2}{*}{2.5} & 5 & 0.39 & 0.37 & 0.33 & 5 & 18 & 56 & 67 & 73 & -16 & -23 \\
\hline & 10 & 0.40 & 0.40 & 0.40 & 0 & 0 & 57 & 68 & 73 & -16 & -22 \\
\hline \multirow{2}{*}{5} & 5 & 0.26 & 0.30 & 0.22 & -13 & 18 & 113 & 132 & 144 & -14 & -22 \\
\hline & 10 & 0.40 & 0.40 & 0.38 & 0 & 5 & 116 & 136 & 144 & -15 & -19 \\
\hline \multirow{2}{*}{7.5} & 5 & 0.24 & 0.32 & 0.18 & -25 & 33 & 190 & 190 & 199 & 0 & -5 \\
\hline & 10 & 0.25 & 0.38 & 0.31 & -34 & -19 & 174 & 190 & 191 & -8 & -9 \\
\hline
\end{tabular}


of higher velocities. However, the results obtained by the three mentioned models show good agreement in general. The main advantage of the simplified helmet model is the fact that the total computational time was 200-times lower than in the case of the full FE helmet model.

\section{Discussion}

The simplified approach is validated using the oblique impact scenarios in 4 impact directions, where the combination of 3 normal with 2 tangential velocities $(4 \times 3 \times 2$ validation matrix $)$ is taken into account. The performance of the simplified helmet model shows similar response concerning the maximum of the relation between the tangential and the normal contact forces, the head acceleration response and the maximum head acceleration.

The average calculation time for the rigid body helmet model is $35 \mathrm{~s}$, whilst the average calculation time for the full FE helmet model is $6262 \mathrm{~s}$. Thus, the application of the rigid body approach makes the calculation approximately 180-times faster. So the main advantage of the approach using the simplified helmet is considerable reduction of the computational time.

The first limitation of the rigid body helmet model is the fact that the validation is provided with the help of another virtual model. However, the THUMS model is widely used and validated for many impact scenarios, so its biofidelity and response are considered as approved [12]. The second limitation of the study is hidden in the simple 3D nonlinear spring joint linking the simplified helmet to the head. Additionally, the rotational stiffness is neglected, but the validation approved this assumption at this stage. Due to the limited spring material model in the used computational environment, the energy absorption is modelled using an exponential unloading curve. However, such approach is fully suitable for the optimization simulation related to the powered two wheelers (PTW) safety and the injury assessment, where the helmet is a necessary part of the model and the loading phase of the impact is the major factor influencing the head injury risk.

\section{Conclusions}

The TW riders belong to the most injured VRU in the case of traffic accidents. With the increasing number of cyclists and the PTW riders for both leisure and/or travelling to work, the injury risk assessment plays an important role. For this case, the mass and the size of the rider is comparable to the mass and the size of the used vehicle. Hence the rider highly influences the vehicle behavior, but at the same time he/she also suffers considerable injuries due to the limited protection.

Due to the reason mentioned above, the PPE plays an important role. Not only a helmet, which is compulsory in many countries, but also the additional protective equipment like energy absorbers and special suits are being developed. For their optimization by virtual prototyping and for describing the complex human body behavior during the complex loading, the helmet is crucial, but it is not necessary to model it in high detail.

The above numerical study shows that the simplified helmet model linked to the human MBS as an additional rigid body leads to the same values regarding the impact response measured by the maximum of the relation between the maximum tangential and normal contact forces and the maximum head acceleration. The benefit of the developed model is the considerable decrease in the required computational time. The results suggest that a similar approach can be applied to the human model wearing a bicycle helmet for the safety assessment and the optimization of the other TW riders. 


\section{Acknowledgements}

The work has been supported by the research project LTC17001 "Exploitation of virtual human model for reducing injury risk of PTW riders" as the national link to the COST Action TU1407 "Scientific and technical innovations for safer Powered Two Wheelers (PTW)" and the internal grant project SGS-2016-059 "Computer modelling and monitoring of human body used for medicine".

\section{References}

[1] Bońkowski, T., Hynčík, L., Šoltés, L., Motorcycle helmets: The population diversity influence on head injury criterion assessment, Proceedings of IRCOBI Conference, 2017, pp. 218-219.

[2] COST327, Motorcycle safety helmets, Final Report of the Action, Belgium, 2001.

[3] ECE 22.05, E/ECE/324 \& E/ECE/TRANS/505 Addendum 21, Regulation No. 22, Revision 4, 2002.

[4] ERSO, Annual Accident Report 2016, Available at (25/10/2017): https://ec.europa.eu/transport/road_safety/sites/roadsafety/files/pdf/statistics/dacota/asr2016.pdf.

[5] Ghajari, M., Galvanetto, U., Iannucci, L., Willinger, R., Influence of the body on the response of the helmeted head during impact, International Journal of Crashworthiness 16 (3) (2011) 285-295. https://doi.org/10.1080/13588265.2011.559798

[6] Ghajari, M., Peldschus, S., Galvanetto, U., Iannucci, L., Effects of the presence of the body in helmet oblique impacts, Accident Analysis and Prevention 50 (2013) 263-271. https://doi.org/10.1016/j.aap.2012.04.016

[7] Hynčík, L., Čechová, H., Kováŕ, L., Bláha, P., On scaling virtual human models, SAE Technical Paper 2013-01-0074 (2013). https://doi.org/10.4271/2013-01-0074

[8] Khosroshahi, S. F., Ghajari, M., Galvanetto, U., A numerical approach for the optimisation of a composite chin bar for protection against basilar skull fracture, Proceedings of IRCOBI Conference, Malaga, 2016, pp. 383-384.

[9] Milne, G., Deck, C., Bourdet, N., Allinne, Q., Gallego, A., Carreira, R., Willinger, R., Assessment of bicyclist head injury risk under tangential impact conditions, Proceedings of IRCOBI Conference, Gothenburg, 2013, pp. 735-746.

[10] Östh, J., Mendoza-Vazquez, M., Linder, A., Svensson, M., Brolin, K., The VIVA OpenHBM finite element 50th percentile female occupant model: Whole body model development and kinematic validation, Proceedings of IRCOBI Conference, Antwerp, 2017, pp. 443-466.

[11] Rigby, P., Juhas, B., Wong, J., Chan, P., Evaluation of biofidelity of ECE Regulation No. 22 injury criteria, Proceedings of 22nd Enhanced Safety of Vehicles Conference, Washington, 2011, pp. 1-16.

[12] THUMS, Am50 pedestrian model: Version 3, 0-080225, Toyota Central R\&D Labs, 2008.

[13] Vychytil, J., Maňas, J., Čechová, H., Špirk, S., Hynčík, L., Kovář, L., Scalable multi-purpose virtual human model for future safety assessment, SAE Technical Paper 2014-01-0534 (2014). https://doi.org/10.4271/2014-01-0534

[14] WHO, Motorcycle helmet laws, by occupant - Data by country, Available at (12/01/2015): http://apps.who.int/gho/data/view.main.51427. 